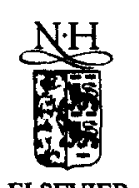

\title{
An inquiry into anatomy of conflicts ${ }^{1}$
}

\author{
Zdzisław Pawlak ${ }^{2}$ \\ Institute of Theoretical and Applied Informatics, Polish Academy of Sciences, ul. Baltycka 5. \\ 44000 Gliwice, Poland
}

Received 1 February 1997; received in revised form 20 August 1997; accepted 20 October 1997

\begin{abstract}
Conflict analysis and resolution play an important role in business, governmental, political and lawsuit disputes, labor-management negotiations, military operations and others. Many mathematical models of conflict situations have been proposed and investigated. In this paper a novel approach to conflict analysis, based on rough set theory, is outlined. Basic concepts of this approach are defined and analyzed. An illustration of the introduced concepts by the Middle East conflict is presented. (c) 1998 Elsevier Science Inc. All rights reserved.
\end{abstract}

Keywords: Conflict analysis; Conflict resolution; Decision analysis; Rough sets

\section{Introduction}

It is a great honor and pleasure for me to receive the JCIS'97 Fourth Annual Lotfi A. Zadeh Best Paper Award.

This paper is devoted to conflict analysis. There are at least two reasons for choosing this topic for this special occasion.

Firstly, conflicts are, no doubt, one of the most characteristic attributes of human nature and study of conflicts is of utmost importance both practically and theoretically. Conflict analysis and resolution play an important role in business, governmental, political and lawsuits disputes, labor-management

\footnotetext{
'This paper is the Lotfi A. Zadeh Best Paper Award's acceptance lecture duing JCIS 1997 delivered on 4 March 1997.

${ }^{2}$ E-mail: zpw@ii.pw.edu.pl.
} 
negotiations, military operations and others. To this end formal models of conflict situations are necessary. Some of them can be found in [e.g., 4,6,12,15-17]. It seems that fuzzy and rough sets are perfect candidates for modeling conflict situations in the presence of uncertainty, but to my knowledge not very much has been done in this area so far, particularly when fuzzy sets are concerned.

Secondly, conflict analysis also seems to be important to data mining. Basic issue in data mining is discovering patterns in data. Usually we are interested in this case in searching for "cause-effect" relations. However in conflict analysis we are interested in another kind of relations, namely conflict, neutrality and alliance relations. In fact we are interested here rather in approximate, then crisp relations, thus fuzzy and rough sets seem to be particularly suited to do this task.

In this paper I would like to outline basic ideas of conflict theory, based on author's previous works $[12,15,16]$ and pursued by many authors [e.g., $3,7,9,10,21-30]$.

The aim of this paper is to call attention of this community to this new, challenging area.

\section{What are conflicts?}

In a conflict at least two parties, called agents, are in dispute over some issues. A very simple conflict is presented in Fig. 1. In this conflict agents are two

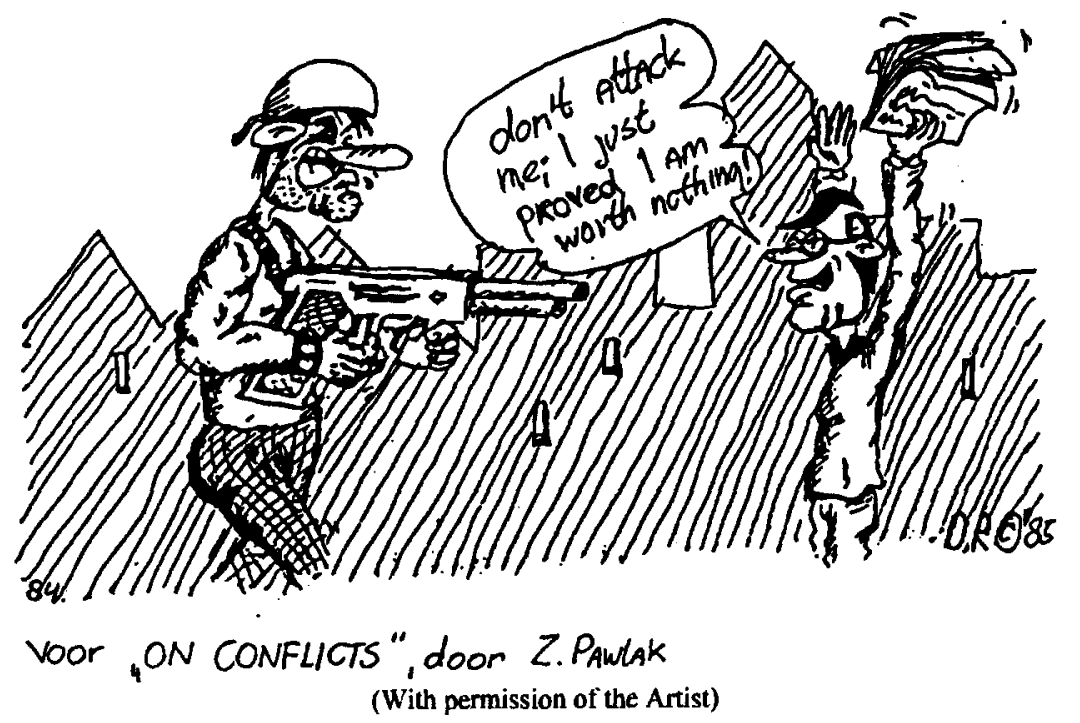

Fig. 1. Artist's vision of a conflict. 
individuals. In general the agents may be individuals, groups, companies, states, political parties etc.

Before we start formal considerations let us first consider an example of the Middle East conflict, which is taken with slight modifications from [1].

The example does not necessarily reflect present-day situation in this region but is used here only as an illustration of the basic ideas considered in this paper.

In this example there are six agents

1. Israel,

2. Egypt,

3. Palestinians,

4. Jordan,

5. Syria,

6. Saudi Arabia, and five issues:

a autonomous Palestinian state on the West Bank and Gaza

$b \quad$ Israeli military outpost along the Jordan River

c Israeli retains East Jerusalem

$d \quad$ Israeli military outposts on the Golan Heights

$e \quad$ Arab countries grant citizenship to Palestinians who choose to remain within their borders

The relationship of each agent to a specific issue can be clearly depicted in the form of a table, as shown in Table 1.

In the table the attitude of six nations of the Middle East region to the above issues is presented: -1 means, that an agent is against, 1 means favorable and 0 neutral toward the issue. For the sake of simplicity we will write - and + instead of -1 and 1 , respectively.

Each row of the table characterizes uniquely an agent, by his approach to the disputed issues.

In conflict analysis primarily we are interested in finding the relationship between agents taking part in the dispute, and investigate what can be done in order to improve the relationship between agents, or in other words how the conflict can be resolved.

Table 1

Information system for the Middle East conflict

\begin{tabular}{llllll}
\hline$U$ & $a$ & $b$ & $c$ & $d$ & $e$ \\
\hline 1 & - & + & + & + & + \\
2 & + & 0 & - & - & - \\
3 & + & - & - & - & 0 \\
4 & 0 & - & - & 0 & - \\
5 & + & - & - & - & - \\
6 & 0 & + & - & 0 & + \\
\hline
\end{tabular}




\section{Conflict, alliance and neutrality}

Tables as shown in Section 2 are known as information systems. An information system is a table rows of which are labeled by objects (agents), columns by attributes (issues) and entries of the table are values of attributes (opinions, beliefs, views, votes, etc.), which are uniquely assigned to each agent and an attribute, i.e. each entry corresponding to row $x$ and column $a$ represents opinion of agent $x$ about issue $a$.

Formally an information system can be defined as a pair $S=(U, A)$, where $U$ is a nonempty, finite set called the universe; elements of $U$ will be called objects (agents), and $A$ is a nonempty, finite set of attributes (issues).

Every attribute $a \in A$ is a total function $a: U \rightarrow V_{a}$, where $V_{a}$ is the set of values of $a$, called the domain of $a$; elements of $V_{a}$ will be referred to as opinions, and $a(x)$ is opinion of agent $x$ about issue $a$.

The above given definition is general, but for conflict analysis we will need its simplified version, where the domain of each attribute is restricted to three values only, i.e. $V_{a}=\{-1,0,1\}$, for every a, meaning against, neutral and favorable, respectively. For the sake of simplicity we will assume $V_{a}=\{-, 0,+\}$. Every information system with the above said restriction will be referred to as a situation.

An information system contains explicit information about the attitude of each agent to issues being considered in the debate, and will be used to derive various implicit information, necessary to conflict analysis.

In order to express relations between agents we define three basic binary relations on the universe: conflict, neutrality and alliance. To this end we need the following auxiliary function:

$$
\phi_{a}(x, y)= \begin{cases}1 & \text { if } a(x) a(y)=1 \quad \text { or } \quad x=y \\ 0 & \text { if } a(x) a(y)=0 \quad \text { and } x \neq y \\ -1 & \text { if } a(x) a(y)=-1\end{cases}
$$

This means that, if $\phi_{a}(x, y)=1$, agents $x$ and $y$ have the same opinion about issue $a$ (are allied on $a$ ); if $\phi_{a}(x, y)=0$ means that at least one agent $x$ or $y$ has neutral approach to issue $a$ (is neutral on $a$ ), and if $\phi_{a}(x, y)=-1$, means that both agents have different opinions about issue $a$ (are in conflict on $a$ ).

In what follows we will define three basic relations $R_{a}^{+}, R_{a}^{0}$ and $R_{a}^{-}$over $U^{2}$ called alliance, neutrality and conflict relations respectively, and defined as follows:

$$
\begin{aligned}
& R_{a}^{+}(x, y) \text { iff } \phi_{a}(x, y)=1, \\
& R_{a}^{0}(x, y) \text { iff } \phi_{a}(x, y)=0 \\
& R_{a}^{-}(x, y) \text { iff } \phi_{a}(x, y)=-1 .
\end{aligned}
$$


It is easily seen that the alliance relation has the following properties:

(i) $R_{a}^{+}(x, x)$,

(ii) $R_{a}^{+}(x, y)$ implies $R_{a}^{+}(y, x)$,

(iii) $R_{a}^{+}(x, y)$ and $R_{a}^{+}(y, z)$ implies $R_{a}^{+}(x, z)$,

i.e., $R_{a}^{+}$is an equivalence relation for every $a$. Each equivalence class of alliance relation will be called coalition on $a$. Let us note that the condition (iii) can be expressed as "friend of my friend is my friend".

For the conflict relation we have the following properties:

(iv) non $R_{a}^{-}(x, x)$,

(v) $R_{a}^{-}(x, y)$ implies $R_{a}^{-}(y, x)$,

(vi) $R_{a}^{-}(x, y)$ and $R_{a}^{-}(y, z)$ implies $R_{a}^{+}(x, z)$,

(vii) $R_{a}^{-}(x, y)$ and $R_{a}^{+}(y, z)$ implies $R_{a}^{-}(x, z)$.

Conditions (vi) and (vii) refer to well-known sayings "enemy of my enemy is my friend" and "friend of my enemy is my enemy".

For the neutrality relation we have:

(viii) non $R_{a}^{0}(x, x)$,

(ix) $R_{a}^{0}(x, y)=R_{a}^{0}(y, x)$ (symmetry).

Let us observe that in the conflict and neutrality relations there are no coalitions.

The following property holds $R_{a}^{+} \cup R_{a}^{0} \cup R_{a}^{-}=U^{2}$ because if $(x, y) \in U^{2}$ then $\Phi_{a}(x, y)=1$ or $\Phi_{a}(x, y)=0$ or $\Phi_{a}(x, y)=-1$ so $(x, y) \in R_{a}^{+}$or $(x, y) \in R_{a}^{0}$ or $(x, y) \in R_{a}^{-}$. All the three relations $R_{a}^{+}, R_{a}^{0}$ and $R_{a}^{-}$are pairwise disjoint, i.e., every pair of objects $(x, y)$ belonged to exactly one of the above defined relations (is in conflict, is allied or neutral).

For example in the Middle East conflict, Egypt, Palestinian and Syria are allied on issue $a$ (autonomous Palestinian state on the West Bank and Gaza), Jordan and Israel are neutral to this issue whereas, Israel and Egypt, Israel and Palestinian, and Israel and Syria are in conflict about this issue.

This can be easily illustrated by a graph as shown in Fig. 2 .

Vertices of the graph are labelled by agents, whereas branches of the graph are representing relation between agents. Solid lines are denoting conflicts, dotted line - alliance, and neutrality, for simplicity, is not shown explicitly in the graph.

Remark. It is interesting that the above considerations on conflicts can be interpreted in Euclidean geometry [7]. Suppose that the universe is the set of all straight lines in Euclidean geometry of two dimensions. Then the alliance, conflict and neutrality relations can be interpreted as parallelisms, orthogonality and intersection (at an angle which is neither zero nor right) of straight lines - whereas properties (i)-(ix) can be understood as axioms of plane Euclidean geometry of straight lines (what about Bolayi-Lobaczwski and Rieman geometry?). 


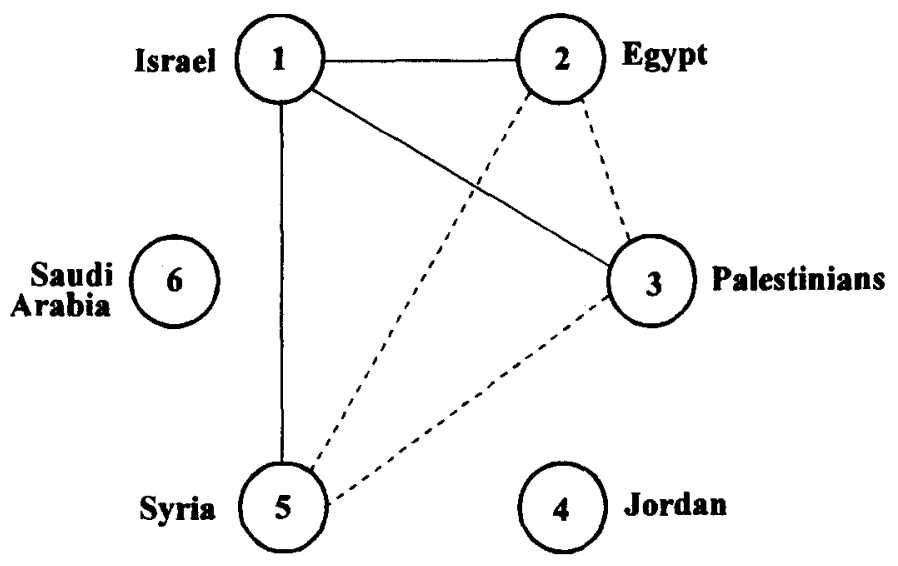

Fig. 2. Graph of the Middle East conflict.

\section{Coalitions}

Let $a \in A$. If there exists a pair $(x, y)$ such that $R_{a}^{-}(x, y)$ we say that the attribute $a$ is conflicting (agents), otherwise the attribute is conflictless. The following property is obvious.

If $a$ is an conflicting attribute, then the relation $R_{a}^{+}$has exactly two equivalence classes $X_{a}^{+}$and $X_{a}^{-}$, where $X_{a}^{+}=\{x \in U: a(x)=+\}, X_{a}^{-}=\{x \in U: a(x)=-\}$, $X_{a}^{0}=\{x \in U: a(x)=0\}$ and $X_{a}^{+} \cup X_{a}^{-} \cup X_{a}^{0}=U$. Moreover $R_{a}^{-}(x, y)$ iff $x \in X_{a}^{+}$ and $y \in X_{a}^{-}$for every $x, y \in U$.

The above proposition says that if $a$ is a conflicting attribute, then all agents are divided into two coalitions (blocks) $X_{a}^{+}$and $X_{a}^{-}$. Any two agents belonging to two different coalitions are in conflict, and the remaining (if any) agents are neutral to the issue $a$.

It follows from the proposition that the graph shown in Fig. 2 can be presented as shown in Fig. 3.

Similar graphs can be given for the remaining attributes.
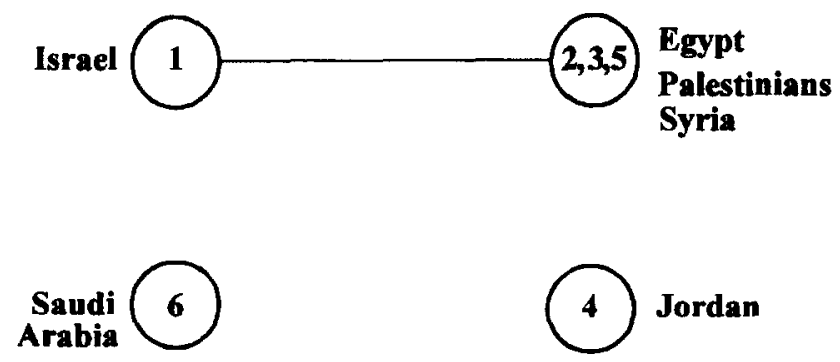

Fig. 3. Simplified graph of the Middle East conflict. 
Table 2

Discernibility matrix for the Middle East conflict

\begin{tabular}{|c|c|c|c|c|c|c|}
\hline & 1 & 2 & 3 & 4 & 5 & 6 \\
\hline \multicolumn{7}{|l|}{1} \\
\hline 2 & $a, b, c, d, e$ & & & & & \\
\hline 3 & $a, b, c, d, e$ & $b, e$ & & & & \\
\hline 4 & $a, b, c, d, e$ & $a, b, d$ & $a, d, e$ & & & \\
\hline 5 & $a, b, c, d, e$ & $b$ & $e$ & $a, d$ & & \\
\hline 6 & $a, c, d$ & $a, b, e, d$ & $a, b, d, e$ & $b, e$ & $a, b, d, e$ & \\
\hline
\end{tabular}

\section{Dissimilarities between agents}

In order to study the differences between agents we can also use a concept of a discernibility matrix [20].

Let $S=(U, A), B \subseteq A$. By a discernibility matrix of $B$ in $S$, denoted $M_{S}(B)$, or $M(B)$, if $S$ is understood, we will mean $n \times n, n=|U|$, matrix defined thus:

$$
\delta_{B}(x, y)=\{a \in B: a(x) \neq a(y)\} .
$$

Thus entry $\delta_{B}(x, y)$, in short, $\delta(x, y)$, is the set of all attributes which discern objects $x$ and $y$.

The discernibility matrix for conflict presented in Table 2 is given below. Each entry of the table shows all issues for which the corresponding agents have different opinions.

The discernibility matrix $M(B)$ assigns to each pair of objects $x$ and $y$ a subset of attributes $\delta(x, y) \subseteq B$, with the following properties:

(i) $\delta(x, x)=\emptyset$,

(ii) $\delta(x, y)=\delta(y, x)$,

(iii) $\delta(x, z) \subseteq \delta(x, y) \cup \delta(y, z)$.

Property (iii) results from the following reasoning. Let $a \notin \delta(x, y) \cup \delta(z, y)$. Hence $a(x)=a(z)$ and $a(z)=a(y)$, so $a(x)=a(y)$. We have $a \notin \delta(x, y)$.

The above properties resemble the well-known properties of distance in a metric space, therefore $\delta$ may be regarded as qualitative metric and $\delta(x, y)$ as qualitative distance.

\section{Degree of conflict}

The relations $R_{a}^{+}, R_{a}^{0}$ and $R_{a}^{-}$can be seen as a description of views on an issue $a$ between agents $x$ and $y$. We will also need an evaluation of views between $x$ and $y$ with respect to the set of issues $B \subseteq A$. To this end we define a function $\rho_{B}(x, y)$ called a conflict function and defined as shown below:

$$
\rho_{B}(x, y)=\frac{\left|\delta_{B}(x, y)\right|}{|B|} .
$$


Obviously $0 \leqslant \rho_{B}(x, y) \leqslant 1$. If $\rho_{B}(x, y) \neq 0$ we will say that $x$ and $y$ are in conflict over $B$ in a degree $\rho_{B}(x, y)$, and of course if $\rho_{B}(x, y)=0, x$ and $y$ are in coalition over $B$.

From definitions of $\rho_{B}, R_{a}^{+}, R_{a}^{-}, R_{a}^{0}$ follows that $\rho_{B}(x, y)=0$, implies $R_{a}^{+}(x, y)$ for every $a \in B$ and $\rho_{B}(x, y)=1$, implies $R_{a}^{-}(x, y)$ for every $a \in B$.

For simplicity we omit $B$ and write $\rho(x, y)$ instead of $\rho_{B}(x, y)$.

The distance function can be used to define approximate (rough) conflict relation $R^{\rho}$, in the following way

$$
R^{\rho}(x, y) \text { iff } \rho(x, y)>0 .
$$

If $R^{\rho}(x, y)$ we say that $x$ and $y$ are in conflict to the degree $\rho(x, y)$.

The following properties are obvious:

1. $\rho(x, x)=0$,

2. $\rho(x, y)=\rho(y, x)$,

3. $\rho(x, z) \leqslant \rho(x, y)+\rho(y, z)$, thus the $\rho(x, y)$ is the distance between $x$ and $y$.

Observe that the condition (3) corresponds to conditions (iii), (vi) and (vii).

For example for the considered Middle East situation the conflict function is shown in Table 3.

The matrix represents a "rough" conflict binary relation. Any entry of the matrix corresponding to elements $x, y$ gives the degree of conflict between $x$ and $y$.

Instead of function $\rho$ we can define function $\rho^{*}$, which defines distance between agents more precisely, by assuming that distance between agents being in conflict is greater than distance between agents which are neutral, i.e.,

$$
\rho_{B}^{*}(x, y)=\frac{\sum_{a \in B} \phi_{a}^{*}(x, y)}{|B|},
$$

where

$$
\phi_{a}^{*}(x, y)=\frac{1-\phi_{a}(x, y)}{2}= \begin{cases}0 & \text { if } a(x) a(y)=1 \text { or } x=y \\ 0.5 & \text { if } a(x) a(y)=0 \\ 1 & \text { if } a(x) a(y)=-1 \text { and } x \neq y\end{cases}
$$

Table 3

Conflict function for the Middle East conflict

\begin{tabular}{lllllll}
\hline & 1 & 2 & 3 & 4 & 5 & 6 \\
\hline 1 & & & & & & \\
2 & 1 & 0.4 & & & \\
3 & 1 & 0.6 & 0.6 & & \\
4 & 1 & 0.2 & 0.2 & 0.4 & \\
5 & 1 & 0.8 & 0.8 & 0.4 & 0.8 \\
6 & 0.6 & 0.8 & & & \\
\hline
\end{tabular}


Table 4

Distance function for the Middle East conflict

\begin{tabular}{lllllll}
\hline & 1 & 2 & 3 & 4 & 5 & 6 \\
\hline 1 & & & & & & \\
2 & 0.9 & & & & & \\
3 & 0.9 & 0.2 & & & & \\
4 & 0.8 & 0.3 & 0.3 & 0.2 & & \\
5 & 1.0 & 0.1 & 0.1 & 0.4 & 0.6 & \\
6 & 0.4 & 0.5 & 0.5 & 0.4 & \\
\hline
\end{tabular}

Employing this distance function for the Middle East conflict we get Table 4. Certainly, this distance function reflects more exactly differences between views of agents than the previous one.

\section{Conflict space}

The concepts discussed in the previous sections can be formulated in a more general way, without referring to the specific issues being debated by agents.

Let $U$ be a nonempty set called the universe and let $\rho$ be a function

$$
\rho: U^{2} \rightarrow[0,1]
$$

such that

1. $\rho(x, x)=0$,

2. $\rho(x, y)=\rho(y, x)$,

3. $\rho(x, z) \leqslant \rho(y, z)+\rho(x, z)$,

for every $x, y, z \in U$.

Obviously $(U, \rho)$ is a metric space, and $\rho(x, y)$ is the distance between $x$ and $y$ in this space.

In the sequel any pair $(U, \rho)$ will be referred to as a conflict space and $\rho(x, y)$ will be called a degree of conflict between $x$ and $y$ in the conflict space.

A pair $x, y$ is said to be:

(i) allied, if $\rho(x, y)<0.5$,

(ii) in conflict, if $\rho(x, y)>0.5$,

(iii) neutral, if $\rho(x, y)=0$.

If $x$ and $y$ are allied, in conflict or are neutral we will write $R^{+}(x, y), R^{-}(x, y)$ or $R^{0}(x, y)$, respectively.

Using the definitions given above conflict presented in Table 4 can be depicted as a graph shown in Fig. 4.

Now the definition of a coalition given in Section $\mathbf{4}$ can be modified as follows.

Set $X \subseteq U$ is a coalition on $B \subseteq A$ if for every $x, y \in U, R^{+}(x, y)$ and $x \neq y$.

In conflict presented in Fig. 4 we have two coalitions: $X_{1}=\{1,6\}$ and $X_{2}=\{2,3,4,5\}$. However in contrast to the proposition given in Section 4, 


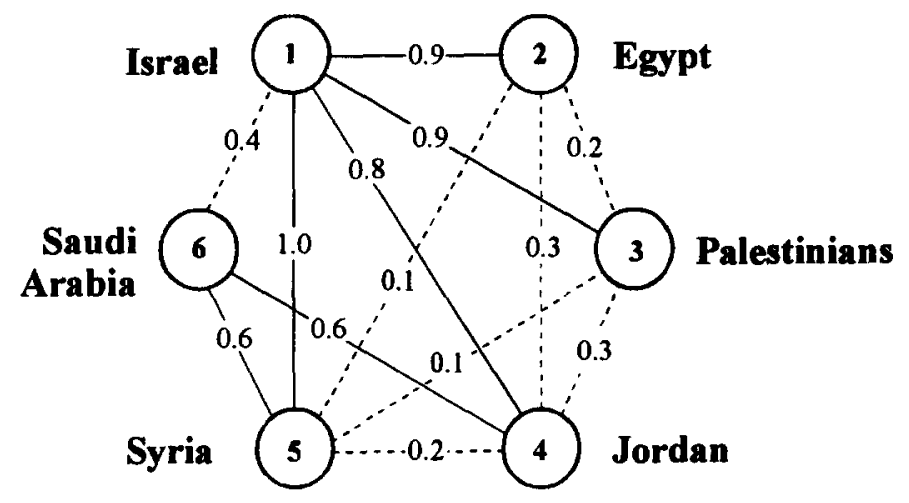

Fig. 4. Example of a generalized conflict.

now the coalitions do not necessarily partition agents into blocks being in total conflict. This is illustrated by the following example. Suppose that the Middle East conflict is presented by Table 5 , and the corresponding conflict matrix is shown in Table 6.

Conflict graph for this case is given in Fig. 5.

We see that now we have three coalitions $Y_{1}=\{1,6\}, Y_{2}=\{5,6\}$ and $Y_{3}=\{2,3,4,5\}$.

Table 5

Coalitions in the Middle East conflict

\begin{tabular}{llllll}
\hline$U$ & $a$ & $b$ & $c$ & $d$ & $e$ \\
\hline 1-Israel & - & + & + & + & + \\
2-Egypt & + & 0 & - & - & - \\
3-Palestinians & + & - & - & - & 0 \\
4-Jordan & 0 & - & - & 0 & - \\
5-Syria & + & 0 & - & - & 0 \\
6-S. Arabia & 0 & + & - & 0 & + \\
\hline
\end{tabular}

Table 6

Conflict matrix for the Middle East conflict

\begin{tabular}{lllllll}
\hline & 1 & 2 & 3 & 4 & 5 & 6 \\
\hline 1 & & & & & & \\
2 & 0.9 & & & & & \\
3 & 0.9 & 0.2 & & & & \\
4 & 0.8 & 0.3 & 0.3 & & & \\
5 & 0.8 & 0.2 & 0.2 & 0.4 & 0.4 & \\
6 & 0.4 & 0.5 & 0.5 & 0.6 & 0.6 \\
\hline
\end{tabular}




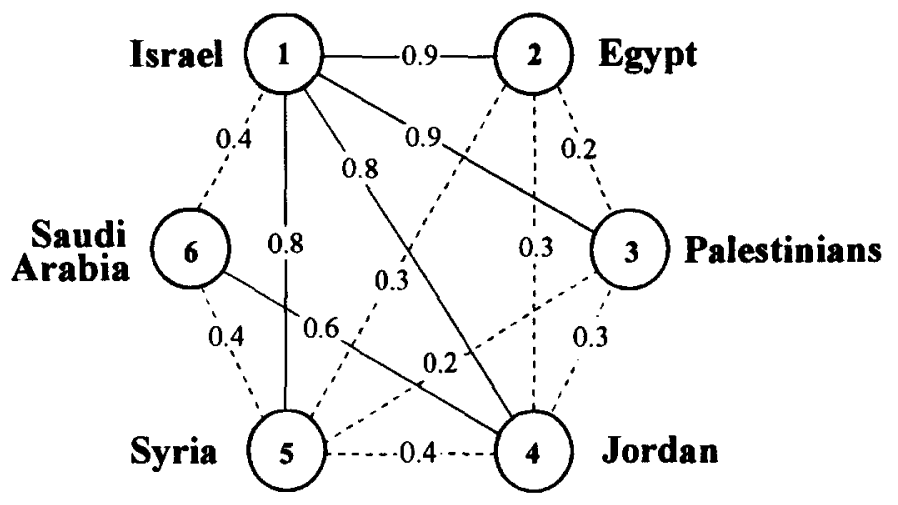

Fig. 5. Another example of a generalized conflict.

We say that coalitions $X, Y \subseteq U$ are strong if $X \cap Y=\emptyset$ and for every $x, y \in U$ such that $x \in X$ and $y \in Y$ non $R^{+}(x, y)$; otherwise the coalitions are weak.

Coalitions $X_{1}=\{1,6\}$ and $X_{2}=\{2,3,4,5\}$ shown in Fig. 4 are strong, whereas all coalitions shown in Fig. 5 are weak.

Let $X$ and $Y$ be strong coalitions. The degree at conflict (tension) between $X$ and $Y$ can be defined by the following formula

$$
\rho^{\prime}(X, Y)=\frac{\sum_{x \in Y, y \in Y, R^{-}(x, y)} \rho(x, y)}{\left|R^{-}(x, y)\right|} .
$$

For example tension between $X_{1}$ and $X_{2}$ in conflict presented in Fig. 4 is $\rho^{\prime}\left(X_{1}, X_{2}\right)=0.8$.

\section{Balance of fear and strategy of intimidation}

In this section we will consider another important problem in the theory of conflicts.

Let $\mu$ be a function

$$
\mu: U \rightarrow[0,+\infty)
$$

which associates to each object $x$ its strength $\mu(x)$.

The strength $\mu(x)$ of $x$ can represent a military or economical potential of the agent.

We assume that each agent should be prepared to fight his enemies and defend himself.

To this end we define a function

$$
\lambda: U^{2} \rightarrow[0,+),
$$

called a strategy, which defines how each agent distributes his forces against his enemies. 
It is reasonable to assume that for every $x, y$ we have:

1. if $\rho(x, y) \leqslant 0.5$, then $\lambda(x, y)=0$,

2. $\sum_{y \in E_{x}} \lambda(x, y) \leqslant \mu(x)$,

where $E_{x}$ is the set of all enemies of $x$, i.e., $E_{x}=\{y \in U: \rho(x, y)>0.5\}$.

One of the most fundamental questions in conflict analysis is if there exists a strategy $\lambda$ called a strategy of intimidation, such that each agent has enough strength to destroy all of his enemies. Formally strategy of intimidation is defined as follows. A strategy $\lambda$ is a strategy of intimidation, if:

1. $\rho(x, y) \geqslant 0.5$ implies $\lambda(x, y)=0$,

2. $\sum_{x \in E_{x}} \hat{\lambda}(x, y)=\mu(x)$,

3. $\lambda(x, y)=\lambda(y, x)$,

for every $x, y \in U$.

The following proposition gives the necessary and suflicient conditions for existence of the strategy of intimidation.

A strategy $\lambda$ is as strategy of intimidation if and only if the system of equations (equations of fear)

$$
\begin{aligned}
& \sum_{y \in E_{x}} \lambda(x, y)=\mu(x), \text { for every } x \in U, \\
& \lambda(x, y)=\lambda(y, x), \text { for every }(x, y) \in U,
\end{aligned}
$$

has a non-negative solution with respect to $\lambda$.

If there exists a strategy of intimidation we say that agents are in a balance of fear.

The examples below illustrate the above result. Consider a conflict situation shown in Fig. 6, where

$$
\mu(x)=3, \mu(y)=4, \mu(z)=5 .
$$

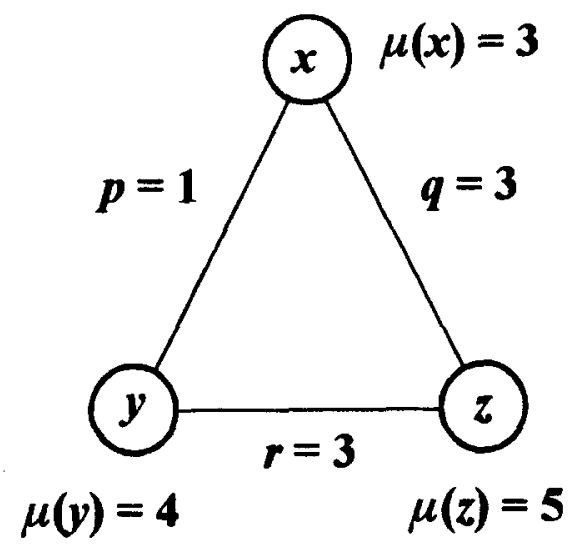

Fig. 6. Strategy of intimidation. 
Let us denote

$$
\begin{aligned}
& \lambda(x, y)=\lambda(y, x)=p, \\
& \lambda(x, z)=\lambda(z, y)=q, \\
& \lambda(y, z)=\lambda(z, y)=r .
\end{aligned}
$$

Then the equations of fear for this situation have the form:

$$
\begin{aligned}
& p+q=3, \\
& p+r=4, \\
& q+r=5 .
\end{aligned}
$$

This system has only one non-negative solution $p=1, q=2, r=3$.

If we assume that $\mu_{z}(x)=3, \mu_{z}(y)=10, \mu_{z}(z)=5$, then the corresponding set of equations has no solution, i.e., a strategy of intimidation does not exist for this conflict situation.

It is easy to give an example of a conflict situation for which we have infinitely many strategies of intimidation.

Many other problems related with conflict analysis and resolution, not presented here, can be easily formulated and solved using the proposed approach.

\section{Conclusion}

The presented attempt to conflict analysis offers deeper insight into structure of conflicts, enables analysis of relationship between parties and issues being debated. It gives many useful clues to support decision making in the presence of conflicts. The presented formal model permits to investigate basic properties of conflict situations and can be used as a basis for mathematical conflict theory. Besides, the mathematical model of conflicts considered here seems especially useful for computer simulation of conflicts, and case based reasoning computer support - in particular when negotiations are concerned.

\section{Acknowledgements}

Thanks are due to the anonymous referee for critical remarks.

\section{References}

[1] J.L. Casti, Alternative Realities - Mathematical Models of Nature and Man, Wiley, New York, 1989. 
[2] C.H. Coombs, G.S. Avrunin, The Structure of Conflict. Lawrence Erlbaum, London, 1988.

[3] R. Deja, Conflict analysis, in: S. Tsumoto, S. Kobayashi, T. Yokomori, H. Tanaka, A. Nakamura, (Eds.), Proceedings of the Fourth International Workshop on Rough Sets, Fuzzy Sets and Machine Discovery, The University of Tokyo, 6-8 November, 1996, pp. 118-124.

[4] P. Dorclan, Interaction under conditions of crisis: Application of graph theory to internation relations, Peace Research Society International Papers 11 (1969) 89-109.

[5] S. French, Decision Theory: An Introduction to the Mathematics of Rationality, Ellis Horwood, Chichester, UK, 1986.

[6] H. Hart, Structures of influence and cooperation-conflict, International Interactions 1 (1974) $141-162$.

[7] A. Kanczewski, On regular conflicts, Bull. Polish Acad. Sci. Math. 33 (11/12) (1985) 685-692.

[8] M. Klein, S.C. Lu, Conflict Resolution in Cooperative Design, International Journal for AI in Engineering 4 (1990) 168-180.

[9] I. Nabialek, Convex sets of balenced strategies in conflict situations, Bull. Polish Acad. Sci. Math. 36 (1988) 425-428.

[10] N.V. Xuat, Security in the theory of conflicts, Bull. Polish Acad. Sci. Math. 32 (1984) 539-541.

[11] H. Nurmi, Comparing Voting Schemes, Reidel, Dordrecht, 1987.

[12] Z. Pawlak, On conflicts, Int. J. Man-Machine Studies 21 (1984) 127-134.

[13] Z. Pawlak, About conflicts (in Polish), Polish Scientic Publishers, Warsaw, 1987, pp. 1-72.

[14] Z. Pawlak, Rough Sets - Theoretical Aspects of Reasoning about Data, Kluwer Academic Publishers, Dordrecht, 1991.

[15] Z. Pawlak, Anatomy of conflicts, Bull. EATCS 50 (1993) 234-246.

[16] Z. Pawlak, On some issues connected with conflict analysis, Institute of Computer Science Reports, 37/93, Warsaw University of Technology (1993).

[17] F. Roberts, Discrete Mathematical Models with Applications to Social, Biological and Environmental Problems, Prentice-Hall, Englewood Cliffs, NJ, 1976.

[18] T.L. Saaty, J. Alexander, Conflict Resolution: The Analytic Hierarchy Process, Praeger, New York, 1989.

[19] T.L. Saaty, War-peace, terrorism and conflict resolution, Manuscript, 1993, pp. 1-22.

[20] A. Skowron, C. Rauszer, The discernibility matrices and functions in information system, in: R. Slowiński (Ed.), Intelligent Decision Support. Handbook of Applications and Advances of the Rough Set Theory, Kluwer Academic Publishers, Dordrecht, 1991, pp. 331-362.

[21] J. Wassowski, Existence of the balanced Strategy in theory of conflicts, Bull. Polish Acad. Sci. Math. 35 (1987) 535-537.

[22] A. Wiweger, On the notation of a conflict, Bull. Polish Acad. Sci. Math. 34 (5/6) (1986) 381-391.

[23] W. Żakowski, On new characterization of regular configurations in theory of conflict situations, Demonstration Mathematica 17 (1984) 211-218.

[24] W. Żakowski, Investigation of balanced situation in theory of conflicts, Bull. Polish Acad. Sci. Math. 32 (7/8) (1984) 379-382.

[25] W. Żakowski, The balanced state in a total-conflict situation, Bull. Polish Acad. Sci. Math. 33 (7/8) (1985) 379-382.

[26] W. Żakowski, On some properties of sets of configurations in theory of conflicts, Bull. Polish Acad. Sci. Math. 34 (1986) 123-126.

[27] W. Żakowski, The balanced strategy in an oriented total-conflict, Bull. Polish Acad. Sci. Math. 35 (1987) 525-530.

[28] W. Zakowski, Safe reduction of conflict in the balanced situations, Bull. Polish Acad. Sci. Math. 36 (1988) 429-435.

[29] W. Żakowski, On some asymmetrical conflicts, Bull. Polish Acad. Sci. Math. 38 (1990) 223228.

[30] W. Żakowski, Sequences of information systems, configurations and conflicts, Bull. Polish Acad. Sci. Tech. Sci. 41 (1993) 297-303. 